\title{
Does conservative kidney management offer a quantity or quality of life benefit compared to dialysis? A systematic review
}

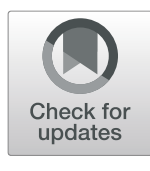

Louise Engelbrecht Buur ${ }^{1,2,3}$, Jens Kristian Madsen', Inge Eidemak ${ }^{4}$, Elizabeth Krarup ${ }^{5}$, Thomas Guldager Lauridsen ${ }^{6}$, Lena Helbo Taasti ${ }^{7}$ and Jeanette Finderup ${ }^{1,2^{*}}$

\begin{abstract}
Background: Patients with stage 5 chronic kidney disease (CKD5) collaborate with their clinicians when choosing their future treatment modality. Most elderly patients with CKD5 may only have two treatment options: dialysis or conservative kidney management (CKM). The objective of this systematic review was to investigate whether CKM offers a quantity or quality of life benefit compared to dialysis for some patients with CKD5.

Methods: The databases MEDLINE, EMBASE, the Cochrane Library, and CINAHL were systematically searched for studies comparing patients with CKD5 who had chosen or were treated with either CKM or dialysis. The primary outcomes were mortality and quality of life (QoL). Hospitalization, symptom burden, and place of death were secondary outcomes. For studies reporting hazard ratios, pooled values were calculated, and forest plots conducted.

Results: Twenty-five primary studies, all observational, were identified. All studies reported an increased mortality in patients treated with CKM (pooled hazard ratio 0.47, $95 \%$ confidence interval 0.34-0.65). For patients aged $\geq 80$ years and for elderly individuals with comorbidities, results were ambiguous. In most studies, CKM seemed advantageous for QoL and secondary outcomes. Findings were limited by the heterogeneity of studies and biased outcomes favouring dialysis.

Conclusions: In general, patients with CKD5 who have chosen or are on CKM live for a shorter time than patients who have chosen or are on dialysis. In patients aged $\geq 80$ years old, and in elderly individuals with comorbidities, the survival benefits of dialysis seem to be lost. Regarding QoL, symptom burden, hospitalization, and place of death, CKM may have advantages. Higher quality studies are needed to guide patients and clinicians in the decision-making process.
\end{abstract}

Keywords: Stage 5 chronic kidney disease, End-stage kidney disease, Dialysis, Conservative kidney management, Mortality, Quality of life

\footnotetext{
* Correspondence: jeajee@rm.dk

'Department of Renal Medicine, Aarhus University Hospital, Palle

Juul-Jensens Boulevard 99, 8200 Aarhus, Denmark

²Department of Clinical Medicine, Aarhus University, Aarhus, Denmark

Full list of author information is available at the end of the article
}

(c) The Author(s) 2021, corrected publication 2022. Open Access This article is licensed under a Creative Commons Attribution 4.0 International License, which permits use, sharing, adaptation, distribution and reproduction in any medium or format, as long as you give appropriate credit to the original author(s) and the source, provide a link to the Creative Commons licence, and indicate if changes were made. The images or other third party material in this article are included in the article's Creative Commons licence, unless indicated otherwise in a credit line to the material. If material is not included in the article's Creative Commons licence and your intended use is not permitted by statutory regulation or exceeds the permitted use, you will need to obtain permission directly from the copyright holder. To view a copy of this licence, visit http://creativecommons.org/ licenses/by/4.0/. The Creative Commons Public Domain Dedication waiver (http://creativecommons.org/publicdomain/zero/1. 0/) applies to the data made available in this article, unless otherwise stated in a credit line to the data. 


\section{Introduction}

Patients with CKD5 have high mortality rates [1]. Most patients are older than 65 years old, and less than $20 \%$ are eligible for a kidney transplant [2].

Many of these older patients with CKD5 are not eligible for a kidney transplant because they are too frail. Therefore, when dialysis is needed, their treatment options are haemodialysis (HD) or peritoneal dialysis (PD), often in the form of assisted automated PD (AAPD). Dialysis can be burdensome for various reasons, including exhausting travel, complications related to the treatment, or the fact that it is so timeconsuming. Thus, while dialysis may prolong patients' lives, it may adversely affect their QoL [3]. For some patients, CKM could be a viable alternative to dialysis. CKM is a treatment strategy that gives patients all the same treatments as those on dialysis, omitting only the dialysis itself [4].

Patients and clinicians may find it challenging to discuss and decide on a future treatment modality based on both the best evidence and the individual patient's preferences. Various aspects have to be considered, which may lead to a complex decision-making process. What is important depends on individual patients and may include survival, QoL, symptom burden, hospitalization, or place of death. Studies of this topic are sparse and heterogeneous, which presents challenges for clinical practice.

Usually, clinical guidelines inform clinical practice [5]. An international guideline from 2012 [6] approaches the structure and process of CKM as an alternative treatment pathway for patients with CKD5 who choose not to pursue kidney replacement therapy. The guideline reports paucity in many of the areas reviewed. However, MEDLINE was the only database searched in establishing this guideline. A European guideline from 2016 [7] addresses the question: What is the benefit of dialysis in frail and older patients? The guideline discusses many important factors related to treatment decision-making such as mortality, QoL, symptom burden, and hospitalization. For some of the issues, however, only a few studies were identified. A more recent UK guideline from 2018 [8] also addresses this question, but includes only mortality as an outcome. Given the lack of clinical guidelines, systematic reviews are beneficial in summarizing the evidence in studies around a specific topic. A number of systematic reviews of varying quality were published at around the same time as the European guideline [9-12]. To date, no randomized controlled trials (RCTs) have been published on this topic.

Recognizing the quality of the European guideline from 2016, the objective of this systematic review was to investigate whether CKM involves quantity or quality of life compared to dialysis for some patients with CKD5 in terms of the outcomes of mortality, QoL, hospitalization, symptom burden, and place of death.

\section{Materials and methods}

The systematic review has been conducted as recommended by the Cochrane Collaboration [13]. The process and results have been documented in accordance with the preferred reporting items for systematic reviews and meta-analyses (PRISMA) statement for reporting systematic reviews $[14,15]$. The protocol for this review was prospectively registered with the Danish Health Authority, and no changes have been made. The review has been conducted by a working group involving doctors and nurses in nephrology as part of the preparation of a national clinical guideline. They have been supported with input from an interprofessional reference group consisting of representatives from the Danish Kidney Association and the Danish professional societies for nephrology, specialized palliative care, geriatrics, and general practitioners.

Eligibility Criteria.

\section{Participants}

Based on the question Does conservative kidney management offer a quantity or quality of life benefit compared to dialysis? this review examined studies including adults aged 18 years old and above who had been diagnosed with CKD5. Studies that included adults with stage 1-4 chronic kidney disease (CKD1-4) or children were excluded.

\section{Interventions}

Studies investigating CKM interventions or any intervention defined as a treatment strategy without dialysis for patients with CKD5 were considered, including patients who had chosen or were receiving CKM.

\section{Comparators}

The review included studies comparing interventions for patients who had chosen or were treated with HD or PD.

\section{Outcomes}

The primary outcomes were mortality and QoL. Secondary outcomes were hospitalization, symptom burden, and place of death, defined as whether the location of death was in accordance with a patient's preference.

\section{Types of Studies Included}

This review considered all study designs relevant for answering the PICO (Patient, Intervention, Comparison, Outcome) question, including secondary literature such as systematic reviews, clinical guidelines, and grey literature. 


\section{Information Sources}

According to the pre-specified protocol, a comprehensive literature search was conducted by the working group in collaboration with a literary search specialist from October 9, 2018 to May 13, 2019. This searched for guidelines in English, Danish, Norwegian, and Swedish published in electronic databases. The databases searched were Guidelines International Network (G-IN), NICE (UK), Scottish Intercollegiate Guidelines Network (SIGN), HTA database, SBU (Sweden), Socialstyrelsen (Sweden), Helsedirektoratet (Norway), Kunnskapssenteret (Norway), MEDLINE, EMBASE, and CINA HL. The databases searched for secondary literature as well as primary literature were MEDLINE, EMBASE, the Cochrane Library, and CINAHL (Additional file 1).

\section{Search Strategy}

A three-phase search strategy was used to locate eligible studies. First, a search for clinical guidelines was conducted, followed by a search for secondary literature (Cochrane reviews, systematic reviews, and metaanalyses). Finally, primary literature was searched without adding any time limitation to our search. The full search protocols are given in the supplementary material (see Additional file 1). The following search terms were used to identify primary studies: exp Kidney Failure, Chronic/ ((end stage or chronic) adj3 (kidney diseas* or kidney failure* or renal disease ${ }^{*}$ or renal failur$\left.\left.\mathrm{e}^{*}\right)\right)$.ab,kf,ti. AND Conservative Treatment/Palliative Care/ ((conservative or supportive) adj3 (treatment* or management or care)).ab,kf,ti. (nondialy* or nondialy*).ab,kf,ti. ((without or refus*) adj2 dialys*) .ab,kf,ti. AND "Quality of Life"/ exp Mortality/Patient Readmission/ ("quality of life" or qol or mortality).ab,kf,ti. place of death.ab,kf,ti. (symptom* adj2 burden*).ab,kf,ti. Life Expectancy/ life expectancy.ab,kf,ti. The search terms 'palliative care' and 'supportive' were included, as these terms by some researchers have been used synonymously with CKM in a broader sense than end-of-life care.

\section{Study Selection}

The process of selecting studies was administered through the systematic review management tool Covidence [16]. Titles were checked for duplicates when entering the eligible literature into Covidence. Two authors independently screened the titles and abstracts of the remaining studies for full-text retrieval. Similarly, two authors independently assessed full-text eligibility for inclusion. Discrepancies in judgement were resolved by consensus.

\section{Data Extraction}

All relevant data and outcomes (mortality, QoL, hospitalization, symptom burden, and place of death) for the PICO question were extracted from each study by two authors independently. Consensus was reached regarding any discrepancies.

\section{Risk of Bias within Studies}

To assess the quality of the studies selected, the Risk Of Bias In Non-randomized Studies of Interventions (ROBINS-I) tool was used because only observational studies were included. Assessment was carried out at outcome level and summarized.

\section{Risk of Bias across Studies}

The Grading of Recommendations Assessment, Development and Evaluation (GRADE) methodology was used to assess the quality of the body of evidence for each relevant outcome in the selected studies [17].

\section{Data Synthesis}

The data extracted from the selected studies was entered into the Review Manager (RevMan5) software [18], used for preparing and updating Cochrane Reviews. Where comparable effect estimates and measures of variance (standard deviation, $95 \%$ confidence intervals) were available, data were pooled using an inverse variance random effect model [19] to conduct a metaanalysis, presented as a forest plot. The overall heterogeneity of the studies was tested using the $\mathrm{I}^{2}$, indicating the variation across studies that is due to heterogeneity, where heterogeneity is indicated by $\mathrm{I}^{2}$ over $75 \%$ [19]. The random effect model was chosen due to an expected heterogeneous effect [19]. Mostly, relevant findings are presented narratively. Means with standard deviations (SD), mean differences, and means with confidence intervals at a $95 \%$ confidence level $(95 \% \mathrm{CI})$ were gathered for continuous data where possible. Hazard ratios (HR), relative risks (RR), and odds ratios (OR) with $95 \% \mathrm{CI}$ were collected from dichotomous data.

\section{Results}

After removal of duplicates, screening of titles and abstracts, and subsequent full-text assessment, a total of one guideline, four systematic reviews, and 25 primary studies were identified. The flow diagram in Fig. 1 gives details of the primary literature search process. Flow diagrams for the guideline search and review search are included in the supplementary material (Additional file 2).

\section{Guideline and Systematic Reviews}

The European guideline [7] covers the evidence relevant for our PICO question until the end of the guideline's literature search in May 2016. All four of the systematic reviews identified $[9,10,12,20]$ synthesized the evidence. Not all the outcomes selected for the PICO 


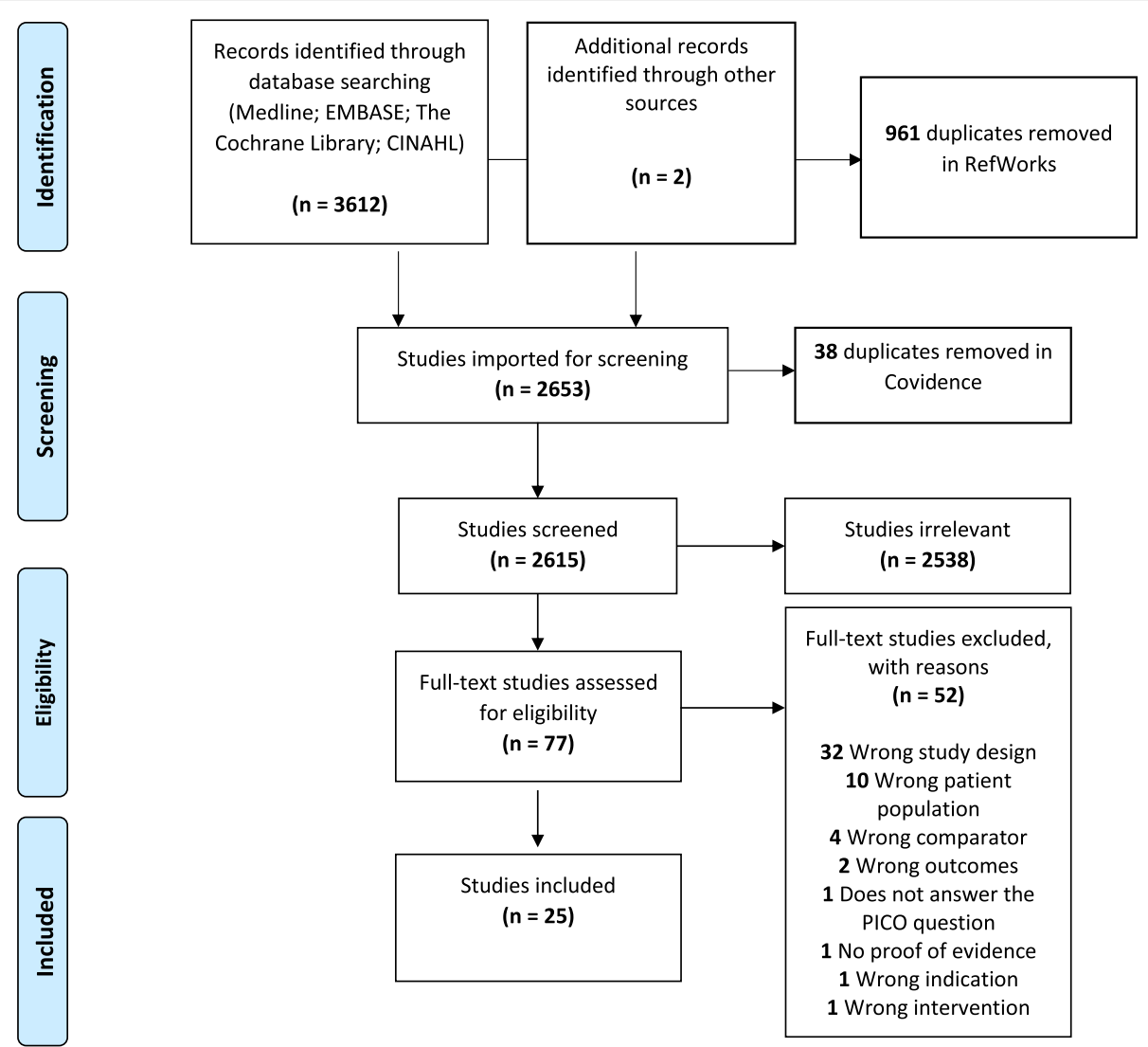

Fig. 1 Flow chart for primary literature search

question in this review were discussed in the earlier reviews. Two studies conducted meta-analyses, one including hospitalization and both including mortality as outcomes $[9,12]$. In the supplementary material, we have provided an overview of the primary studies included in our review compared to studies included in the European guideline and the four systematic reviews selected (Additional file 3). The supplementary material also provides a list of studies present in the guideline or reviews that we did not include, with references and reasons for exclusion (Additional file 4).

\section{Primary Literature}

Table 1 presents basic characteristics and data extraction for the 25 primary studies selected. All studies were observational: 11 prospective cohort studies [3, 21-30]; 10 retrospective cohort studies [31-40]; and 4 cross-sectional studies [41-44]. In general, patients on CKM were older and, when reported, had more comorbidities and poorer functional status compared to other groups. Nine of the included studies were conducted in UK $[3,22,24,25,31,39-41,43]$, four in Hong Kong [28, 36, 38, 44] and three studies in Australia [27, 29, 43] as well as in the Netherlands
[21, 32, 33]. The heterogeneity between studies in relation to interventions, comparators, statistical analyses, and treatment modalities affected the possibility of conducting meta-analyses for all outcomes except mortality and hospitalization. Even for these two outcomes, not all studies reported data suitable for metaanalyses. The quality assessments using ROBINS-I are presented in the supplementary material (Additional file 5), showing that only three studies had a low risk of bias, 12 studies had a moderate risk of bias, nine studies had a serious risk of bias, and one study had a critical risk of bias. The directness of bias for most studies was unpredictable or in favour of dialysis.

\section{Mortality}

In total, 18 of both prospective and retrospective primary observational studies comparing CKM and dialysis, patients on CKM had higher mortality rates [3, 21-26, $28,29,31-40]$. A meta-analysis is presented in Fig. 2. In a study of patients $\geq 75$ years old, the higher mortality rate for those on CKM compared to dialysis was significantly reduced in patients with high comorbidities, especially ischemic heart disease [31]. For patients $\geq 80$ years, the results seem conflicting. One study investigating 
Table 1 Table with Details of the Primary Studies Selected for Inclusion

\begin{tabular}{|c|c|c|c|c|c|}
\hline $\begin{array}{l}\text { Author, } \\
\text { year of } \\
\text { publication, } \\
\text { country }\end{array}$ & Population & Age (years) ${ }^{1}$ & $\begin{array}{l}\text { Intervention, } \\
\mathrm{n}\end{array}$ & $\begin{array}{l}\text { Comparator, } \\
\text { n }\end{array}$ & Main outcome measures and results ${ }^{1}$ \\
\hline $\begin{array}{l}\text { Almutary, } \\
\text { 2016, Saudi } \\
\text { Arabia [42] }\end{array}$ & $\begin{array}{l}\text { Non-dialysis } \\
\text { group (CKD4 \& } \\
\text { CKM (CKD5)) } \\
\text { Dialysis group } \\
\text { (HD \& PD) }\end{array}$ & $\begin{array}{l}\text { Total group, } 48.29( \pm \\
14.86) \\
\text { CKM, (CKD5), } 51.84 \pm \\
15.11 \\
\text { HD, } 47.71 \pm 14.46 \\
\text { PD, } 43.08 \pm 15.09\end{array}$ & $\begin{array}{l}\text { CKM (CKD5), } \\
38\end{array}$ & $\begin{array}{l}\mathrm{HD}, 287 \\
\mathrm{PD}, 42\end{array}$ & $\begin{array}{l}\text { Symptom burden (CKD-SBI) } \\
\text { Dialysis vs. CKM (CKD5): } \\
\text { HD: } 23.36 \pm 16.99 ; \text { PD: } 12.04 \pm 6.58 \text {, vs. CKM (CKD5): } 8.1 \pm \\
8.04 ; p<0.001\end{array}$ \\
\hline $\begin{array}{l}\text { Brown, 2015, } \\
\text { Australia [29] }\end{array}$ & CKD4 \& CKD5 & $\begin{array}{l}\text { CKM, } 82 \pm 9 \\
\text { Pre-dialysis, } 69 \pm 14\end{array}$ & CKM, 122 & $\begin{array}{l}\text { Pre-dialysis, } \\
273 \\
\text { HD, } 55 \\
\text { PD, } 37\end{array}$ & $\begin{array}{l}\text { Mortality } \\
\text { Dialysis vs. CKM, HR: } 0.30 \text { ( } 95 \% \text { Cl: } 0.13-0.67 \text { ); } p=0.003 \text {. } \\
\text { Symptom burden \& Quality of life } \\
\text { No difference over time in groups. }\end{array}$ \\
\hline $\begin{array}{l}\text { Carson, 2009, } \\
\text { UK [24] }\end{array}$ & CKD5 & $\begin{array}{l}\text { Age } \geq 70 \text { at inclusion } \\
\text { CKM, mean } 81.6 ; \\
\text { median } 83 \\
\text { Dialysis, mean } 76.4 ; \\
\text { median } 75\end{array}$ & CKM, 29 & Dialysis, 173 & $\begin{array}{l}\text { Hospitalization rate } \\
\text { Hospital days/patient days survived: } \\
\text { CKM, } 4.3 \pm 0.26 ; \text { RRT, } 6.9 \pm 0.71 \\
\text { Place of death } \\
\text { Home/hospice: CKM, } 40 \% \text {; Dialysis } 21 \% \text {, } \\
\text { Hospital: CKM, } 36 \% \text {; Dialysis } 70 \%\end{array}$ \\
\hline $\begin{array}{l}\text { Chandna, } \\
\text { 2011, UK [40] }\end{array}$ & CKD5 & $\begin{array}{l}\text { CKM, } 77.5 \pm 7.6 \\
\text { Dialysis, } 58.5 \pm 15.0\end{array}$ & CKM, 155 & Dialysis, 689 & $\begin{array}{l}\text { Mortality } \\
\text { Mortality in patients aged > 75: } \\
\text { CKM vs. dialysis, HR: } 1.18 \text { ( } 95 \% \text { Cl: } 0.79-1.76) ; p=0.428 \text {. } \\
\text { Months of survival in patients with comorbidity score > } \\
\text { 4: Dialysis, } 25.8 \pm 4.4(\mathrm{SE}) ; \mathrm{CKM}, 20.4 \pm 2.4(\mathrm{SE}) ; p=0.83 \text {. }\end{array}$ \\
\hline $\begin{array}{l}\text { Da Silva-Gane, } \\
\text { 2012, UK [3] }\end{array}$ & CKD4-5 & $\begin{array}{l}H D, 60.6 \pm 14.9 \\
P D, 48.0 \pm 15.6 \\
C K M, 77.5 \pm 6.5\end{array}$ & CKM, 30 & $\begin{array}{l}\mathrm{HD}, 80 \\
\mathrm{PD}, 44\end{array}$ & $\begin{array}{l}\text { Mortality } \\
\text { HD vs. CKM, HR } 0.47 \text { ( } 95 \% \text { Cl: } 0.20-1.10) ; p=0.08 \\
\text { PD vs. CKM, HR: } 0.39 \text { ( } 95 \% \text { Cl: } 0.10-1.48) ; p=0.17 \\
\text { Quality of life } \\
\text { No difference over time in groups. }\end{array}$ \\
\hline $\begin{array}{l}\text { Hussain, } \\
\text { 2013, UK [39] }\end{array}$ & CKD5 & Age $>70$ at inclusion & CKM, 172 & Dialysis, 269 & $\begin{array}{l}\text { Hospitalization } \\
\text { Dialysis vs. CKM, RR } 1.6 \text { ( } 95 \% \text { Cl: 1.14-2.25), } p<0.05 \text {. }\end{array}$ \\
\hline $\begin{array}{l}\text { lyasere, } \\
2019, \text { UK [41] }\end{array}$ & CKD5 & $\begin{array}{l}\text { Median age (IQR) } \\
\text { CKM, } 83 \text { (80-88) } \\
\text { aAPD, } 81(79-88) \\
\text { HD, } 82(78-85)\end{array}$ & CKM, 28 & $\begin{array}{l}\mathrm{HD}, 28 \\
\mathrm{PD}, 28\end{array}$ & $\begin{array}{l}\text { Quality of life } \\
\text { SF-12, PCS: CKM, } 28.9 \pm 10.0 ; \text { dialysis, } 29.2 \pm 8.3 ; p=0.90 \\
\text { SF-12, MCS: CKM, } 46.3 \pm 12.1 \text {; dialysis, } 49.9 \pm 12.9 ; p=0.28\end{array}$ \\
\hline $\begin{array}{l}\text { Joly, } \\
\text { 2003, France } \\
{[23]}\end{array}$ & CKD4-5 & $\begin{array}{l}\text { Age } \geq 80 \text { at inclusion } \\
\text { CKM, } 84.1 \pm 2.9 \\
\text { Dialysis, } 83.2 \pm 2.9\end{array}$ & CKM, 43 & Dialysis, 101 & $\begin{array}{l}\text { Mortality } \\
\text { CKM, } 8.9 \text { months ( } 95 \% \text { Cl: 4-10); dialysis, } 28.9 \text { months } \\
\text { (95\% Cl: 24-38); } p<0.0001\end{array}$ \\
\hline $\begin{array}{l}\text { Kwok, } \\
\text { 2016, Hong } \\
\text { Kong [38] }\end{array}$ & CKD5 & $\begin{array}{l}\text { Age } \geq 65 \text { at inclusion } \\
\text { CKM, } 79.6 \pm 6.8 \\
\text { Dialysis, } 74.2 \pm 6.1\end{array}$ & CKM, 432 & Dialysis, 126 & $\begin{array}{l}\text { Mortality } \\
\text { CKM, } 10.0 \text { months ( } 95 \% \text { Cl: } 8.3-11.7) \text {; dialysis, } 44.6 \\
\text { months ( } 95 \% \text { Cl: } 37.3-51.9 \text { ); } p<0.001 \text {. }\end{array}$ \\
\hline $\begin{array}{l}\text { Murtagh, } \\
\text { 2007, UK [31] }\end{array}$ & CKD5 & $\begin{array}{l}\text { Age }>75 \text { at inclusion } \\
\text { CKM, } 81.36 \\
\text { Dialyse, } 78,17\end{array}$ & CKM, 77 & Dialysis, 52 & $\begin{array}{l}\text { Mortality } \\
\text { CKM vs. dialysis, HR: } 2.90 \text { ( } 95 \% \text { Cl: } 1.60-5.26) \text {; } \\
\text { In patients with comorbidity score }>2 \text { : Dialysis vs. CKM: } \\
\text { log rank statistic }<0.001 \text {, df } 1, p=0.98 \text {, In patients with } \\
\text { ischaemic heart disease: Dialysis vs. CKM: log rank } \\
\text { statistic } 1.46 \text {, df } 1, p<0.27 \text {. }\end{array}$ \\
\hline $\begin{array}{l}\text { Raman, } \\
\text { 2018, UK [22] }\end{array}$ & CKD5 & $\begin{array}{l}\text { Dialysis, } 78.9 \pm 2.8 \\
\text { CKM, } 83.7 \pm 4.2\end{array}$ & CKM, 81 & Dialysis, 123 & $\begin{array}{l}\text { 1-year survival } \\
\text { Dialysis vs. CKM, OR } 0.38 \text { (95 \% Cl: 0.09-1.60); } p=0.19 \\
\text { Hospitalization } \\
\text { Number of admission days (median (IQR)): } \\
\text { CKM, } 0.8(0.0-8.7) ; \text { dialysis, } 2.2(0.7-14.7) ; p=0.005\end{array}$ \\
\hline $\begin{array}{l}\text { Reindl- } \\
\text { Schwaighofer, } \\
\text { 2017, Austria } \\
{[37]}\end{array}$ & CKD5 & $\begin{array}{l}\text { Age }>65 \text { at inclusion } \\
\text { HD, } 74.06 \pm 5.78 \\
\text { CKM, 81.22 } \pm 7.23\end{array}$ & CKM, 174 & $H D, 8622$ & $\begin{array}{l}\text { Mortality } \\
\text { HD vs. CKM, HR: } 0.23 \text { (95\% Cl: 0.18-0.29); } p<0.001 \text {. }\end{array}$ \\
\hline $\begin{array}{l}\text { Seow, 2013, } \\
\text { Singapore }[30]\end{array}$ & CKD5 & $\begin{array}{l}\text { Median age (IQR): } \\
\text { CKM, } 78 \text { (40-70) } \\
\text { Dialysis, } 71 \text { (50-80) }\end{array}$ & CKM, 63 & Dialysis, 38 & $\begin{array}{l}\text { Quality of life } \\
\text { No difference over time in groups. }\end{array}$ \\
\hline $\begin{array}{l}\text { Shah, } \\
\text { 2019, Australia }\end{array}$ & CKD5 & $\begin{array}{l}\text { Age } \geq 75 \text { at inclusion } \\
\text { Median age (IQR): }\end{array}$ & CKM, 46 & Dialysis, 83 & $\begin{array}{l}\text { Quality of life } \\
\text { Dialysis vs. CKM, adjusted differences in KDQOL-36 scores }\end{array}$ \\
\hline
\end{tabular}


Table 1 Table with Details of the Primary Studies Selected for Inclusion (Continued)

\begin{tabular}{|c|c|c|c|c|c|}
\hline $\begin{array}{l}\text { Author, } \\
\text { year of } \\
\text { publication, } \\
\text { country }\end{array}$ & Population & Age (years) ${ }^{1}$ & $\begin{array}{l}\text { Intervention, } \\
\mathrm{n}\end{array}$ & $\begin{array}{l}\text { Comparator, } \\
\text { n }\end{array}$ & Main outcome measures and results ${ }^{1}$ \\
\hline \& UK [43] & & $\begin{array}{l}\text { Dialysis, } 81 \text { (78-84) } \\
\text { CKM, } 83(81-87) \\
\text { Age } \leq 81, n(\%): \\
\text { Dialysis, } 50(6) \\
\text { CKM, } 19(41) \\
\text { Age }>81, n(\%): \\
\text { Dialysis, } 33(40) \\
\text { CKM, } 27(59)\end{array}$ & & & $\begin{array}{l}\text { (95\% Cl): } \\
\text { KDQOL-burden of disease: }-28.59 \text { (-41.77 to }-15.42) ; p< \\
0.001 \\
\text { KDQOL-symptoms of disease: }-5.93(-14.61 \text { to } 2.73) ; p= \\
0.18 \\
\text { KDQOL-effects of disease: }-16.49 \text { (-25.98 to }-6.99) ; p< \\
0.001\end{array}$ \\
\hline $\begin{array}{l}\text { Shum, } \\
\text { 2014, Hong } \\
\text { Kong }[36]\end{array}$ & CKD5 & $\begin{array}{l}\text { Age } 65-90 \text { at } \\
\text { inclusion } \\
\text { Overall age, } 73.8 \pm 5.4 \\
\text { CKM, } 75.3 \pm 5.7 \\
\text { PD, } 73.4 \pm 5.3\end{array}$ & CKM, 42 & PD, 157 & $\begin{array}{l}\text { Mortality } \\
\text { PD vs. CKM, HR: } 0.46 \text { (95\% Cl 0.31-0.68), } p<0.001 \text {. } \\
\text { Hospitalization } \\
\text { Days per person year, median [IQR] } \\
\text { PD, } 16.17 \text { [6.29-43.32] vs. CKM, } 38.01 \text { [6.75-76.56]; } p= \\
0.03\end{array}$ \\
\hline $\begin{array}{l}\text { Smith, } \\
\text { 2003, UK [25] }\end{array}$ & CKD5 & $\begin{array}{l}\text { CKM (palliative care } \\
\text { population), } 71 \pm 12 \\
\text { Dialysis, } 59 \pm 15\end{array}$ & CKM, 63 & Dialysis, 258 & $\begin{array}{l}\text { Mortality } \\
\text { Dialysis, median survival } 8.3 \text { months; CKM, median } \\
\text { survival } 6.3 \text { months; N.S. } \\
\text { Place of death } \\
\text { Deaths at home or in a hospice: CKM, } 22 \text { of } 34 \text { deaths } \\
\text { ( } 65 \%) \\
\text { Dialysis, } 11 \text { of } 41 \text { deaths }(27 \%) ; p=0.001\end{array}$ \\
\hline $\begin{array}{l}\text { Tam-Tham, } \\
\text { 2018, Canada } \\
{[35]}\end{array}$ & CKD5 & $\begin{array}{l}\text { Age } \geq 65 \text { at inclusion } \\
\text { Age } 65 \text { to }<75(n, \%) \\
\text { Dialysis, } 228(45.6) \\
\text { CKM, } 45(13.3) \\
\text { Age } 75 \text { to }<85(n, \%) \\
\text { Dialysis, } 220(44.0) \\
\text { CKM, } 143(42.3) \\
\text { Age } \geq 85(n, \%) \\
\text { Dialysis, } 52(10.4) \\
\text { CKM, } 150(44.4)\end{array}$ & CKM, 338 & Dialysis, 500 & $\begin{array}{l}\text { Mortality } \\
\text { Dialysis vs. CKM (0-3 years), HR: } 0.56 \text { (95 \% Cl: 0.44-0.71); } \\
p<0.001 \\
\text { Dialysis vs. CKM (after } 3 \text { years), HR: } 1.98 \text { (95\% Cl: } 1.16- \\
\text { 3.37); } p=0.12 \\
\text { Hospitalization } \\
\text { Dialysis vs. CKM, HR: } 1.40 \text { (95\% Cl: 1.16-1.69), } p=0.001\end{array}$ \\
\hline $\begin{array}{l}\text { Tan, } \\
\text { 2017, Australia } \\
{[27]}\end{array}$ & CKD5 & $\begin{array}{l}\text { Age }>65 \text { at inclusion } \\
\text { CKM, } 84 \\
\text { Dialysis, } 73\end{array}$ & CKM, 8 & Dialysis, 12 & $\begin{array}{l}\text { Symptom burden } \\
\text { Change (improvement) in mean symptom POS-score } \\
\text { over } 6 \text { months: CKM, 1.5; dialysis, } 7.58 ; p<0.002 \text {. }\end{array}$ \\
\hline $\begin{array}{l}\text { Teo, } \\
\text { 2010, } \\
\text { Singapore }[26]\end{array}$ & CKD5 & $\begin{array}{l}\mathrm{CKM}, 67.4 \pm 11.8 \\
\mathrm{HD}, 58.7 \pm 12.9\end{array}$ & CKM, 16 & HD, 102 & $\begin{array}{l}\text { Mortality } \\
\text { CKM, HR: } 2.29 \text { ( } 95 \% \text { Cl: } 1.16-4.45) ; H D, H R: 0.59 \text { ( } 95 \% \text { Cl: } \\
\text { 0.33-1.05); } p=0.042\end{array}$ \\
\hline $\begin{array}{l}\text { Teruel, } \\
\text { 2015, Spain } \\
\text { [34] }\end{array}$ & CKD5 & $\begin{array}{l}\text { Median age (IQR) } \\
\text { Dialysis, } 68(54,76) \\
\text { CKM, } 83(78,86)\end{array}$ & CKM, 90 & Dialysis, 142 & $\begin{array}{l}\text { Mortality } \\
\text { CKM, } 8.2 / 100 \text { patient months; Dialysis, } 0.6 / 100 \text { patient } \\
\text { months; } \\
p<0.001\end{array}$ \\
\hline $\begin{array}{l}\text { van Loon, } \\
\text { 2019, the } \\
\text { Netherlands } \\
\text { [21] }\end{array}$ & CKD5 & $\begin{array}{l}\text { Age } \geq 65 \text { at inclusion } \\
\text { CKM, } 82 \pm 6 \\
\text { Dialysis, } 75 \pm 7\end{array}$ & CKM, 89 & Dialysis, 192 & $\begin{array}{l}\text { 12-month survival } \\
\text { CKM vs. dialysis, } \\
\text { HR: } 2.12 \text { ( } 95 \% \text { Cl: } 1.12-4.03) ; p=0.02 \\
\text { In patients < } 80 \text { years old, } \\
\text { HR: } 5.05 \text { ( } 95 \% \text { Cl: } 1.90-13.50) ; p<0.01 \\
\text { In patients } \geq 80 \text { years old, } \\
\text { HR: } 1.30 \text { ( } 95 \% \text { Cl: } 0.58-2.91) ; p=0.53 \\
\text { Six-month quality of life } \\
\text { EQ-5D Index, mean (SE) change within group: } \\
\text { CKM, } 0.047 \text { (0.022); } p<0.01 \\
\text { Dialysis, } 0.026 \text { (0.014); } p=0.10 \\
\text { Between group difference, } p<0.01 \\
\text { Hospitalization } \\
\text { Median number [IQR] of admissions: } \\
\text { CKM, } 1[1-5] \text {; Dialysis, }[1-4] ; p=0.27 \\
\text { Hospitalization } \\
\text { Median number [IQR] of admission days: } \\
\text { Dialysis, } 7 \text { [3-15]; CKM, } 4[2-12] ; p=0.22\end{array}$ \\
\hline $\begin{array}{l}\text { Verberne, } \\
\text { 2018, the }\end{array}$ & CKD5 & $\begin{array}{l}\text { CKM, } 82.6 \pm 4.5 \\
\text { Dialysis, } 76.2 \pm 4.4\end{array}$ & CKM, 126 & Dialysis, 240 & $\begin{array}{l}\text { Mortality } \\
\text { CKM vs. dialysis, HR: } 1.67 \text { (95\% Cl: 1.19-2.35), } p=0.003\end{array}$ \\
\hline
\end{tabular}


Table 1 Table with Details of the Primary Studies Selected for Inclusion (Continued)

\begin{tabular}{|c|c|c|c|c|c|}
\hline $\begin{array}{l}\text { Author, } \\
\text { year of } \\
\text { publication, } \\
\text { country }\end{array}$ & Population & Age (years) ${ }^{1}$ & $\begin{array}{l}\text { Intervention, } \\
\mathrm{n}\end{array}$ & $\begin{array}{l}\text { Comparator, } \\
\text { n }\end{array}$ & Main outcome measures and results ${ }^{1}$ \\
\hline $\begin{array}{l}\text { Netherlands } \\
\text { [33] }\end{array}$ & & & & & $\begin{array}{l}\text { Median [IQR] survival in years in patients } \geq 70 \text { years old: } \\
\text { CKM, 1.3 [0.5-2.5]; dialysis, } 3.1[1.7-6.4] ; p<0.001 \\
\text { Median [IQR] survival in years in patients } \geq 80 \text { years old: } \\
\text { CKM, } 2.3 \text { [1.3-3.7]; dialysis, } 2.9 \text { [1.9-6.0]; } p=0.13 \\
\text { Quality of life } \\
\text { No difference between groups }\end{array}$ \\
\hline $\begin{array}{l}\text { Verberne, } \\
\text { 2016, the } \\
\text { Netherlands } \\
\text { [32] }\end{array}$ & CKD5 & $\begin{array}{l}\text { CKM, } 83 \pm 4.5 \\
\text { Dialysis, } 76 \pm 4.4\end{array}$ & CKM, 107 & Dialysis, 204 & $\begin{array}{l}\text { Mortality } \\
\text { Dialysis vs. CKM, HR: } 0.62 \text { ( } 95 \% \text { Cl: } 0.42-0.92), p=0.02 \text {. } \\
\text { Median [IQR] survival in years in patients } \geq 80 \text { years old: } \\
\text { CKM, 1.4 [0.7-3.0]; dialysis, } 2.1[1.5-3.4] ; p=0.08 \text {. }\end{array}$ \\
\hline $\begin{array}{l}\text { Yong, } \\
\text { 2009, Hong } \\
\text { Kong [44] }\end{array}$ & CKD5 & $\begin{array}{l}\text { CKM, } 73.1 \pm 7.1 \\
\text { Dialysis, } 58.2 \pm 11.4\end{array}$ & CKM, 45 & Dialysis, 134 & $\begin{array}{l}\text { Symptom burden } \\
\text { Number of symptoms } \\
\text { CKM, 8.2 } \pm 3.9 \text {; dialysis } 9.3 \pm 4.7, p=0.243\end{array}$ \\
\hline $\begin{array}{l}\text { Yuen, } \\
\text { 2016, Hong } \\
\text { Kong [28] }\end{array}$ & CKD5 & $\begin{array}{l}\text { CKM, } 76.8 \pm 9.1 \\
\text { Dialysis, } 58.6 \pm 12.6\end{array}$ & CKM, 335 & Dialysis, 265 & $\begin{array}{l}\text { One-year survival (\%) } \\
\text { CKM, } 57.3 \pm 2.9 \text {; dialysis, } 89.7 \pm 2.1 \\
\text { 3-year survival (\%) } \\
\text { CKM, } 16 \pm 2.7 \text {; dialysis, } 74.6 \% \pm 3.4\end{array}$ \\
\hline
\end{tabular}

CKD4 and CKD5 stage 4 and 5 chronic kidney disease; CKM conservative kidney management; HD haemodialysis; $P D$ peritoneal dialysis; $A A P D$ assisted automated peritoneal dialysis; IQR interquartile range; $H R$ hazard ratio; eGFR estimated glomerular filtration rate; SE standard error; RR relative risk; CKD-SBI The CKD Symptom Burden Index; SF-12 short form 12; MCS mental component summary; PCS physical component summary; KDQOL Kidney Disease Quality of Life; POS Palliative Outcome Scale; EQ-5D European Quality of life-5 Dimensions

1 Unless otherwise noted, values are expressed as mean \pm SD

octogenarians reported higher mortality for patients on CKM [23]. In contrast, three other studies found overall that mortality was equal for patients $\geq 80$ years old [21, 32, 33].

\section{Quality of life}

One prospective cohort study found that patients with CKD5 on CKM had poorer self-reported QoL at baseline compared to patients on dialysis, but only with a borderline significance $(p=0.05)$ [21]. At a six-month followup, self-reported QoL was higher among patients on CKM compared to those on dialysis $(p<0.01)$. Another prospective cohort study found no difference in the mental health summary scores at baseline but significant difference in the physical health summary scores $(\mathrm{p}=$ 0.001). There was, however, no difference in QoL over time [3], a finding seen also in two other prospective cohort studies $[29,30]$ In a retrospective cohort study, there were no difference between patients managed conservatively and dialysis patients on physical and mental health summary scores [33]. The results of two crosssectional studies were heterogeneous $[41,43]$. One study found no difference in self-reported QoL (SF-36) between patients on CKM, HD, or PD [41]. Results from the other cross-sectional study varied depending on the tool used to measure QoL.

\section{Symptom burden}

In a small prospective study, commencement of dialysis in a younger cohort of elderly patients was associated with decrease in overall symptom burden [27]. In another prospective cohort study, however, no difference

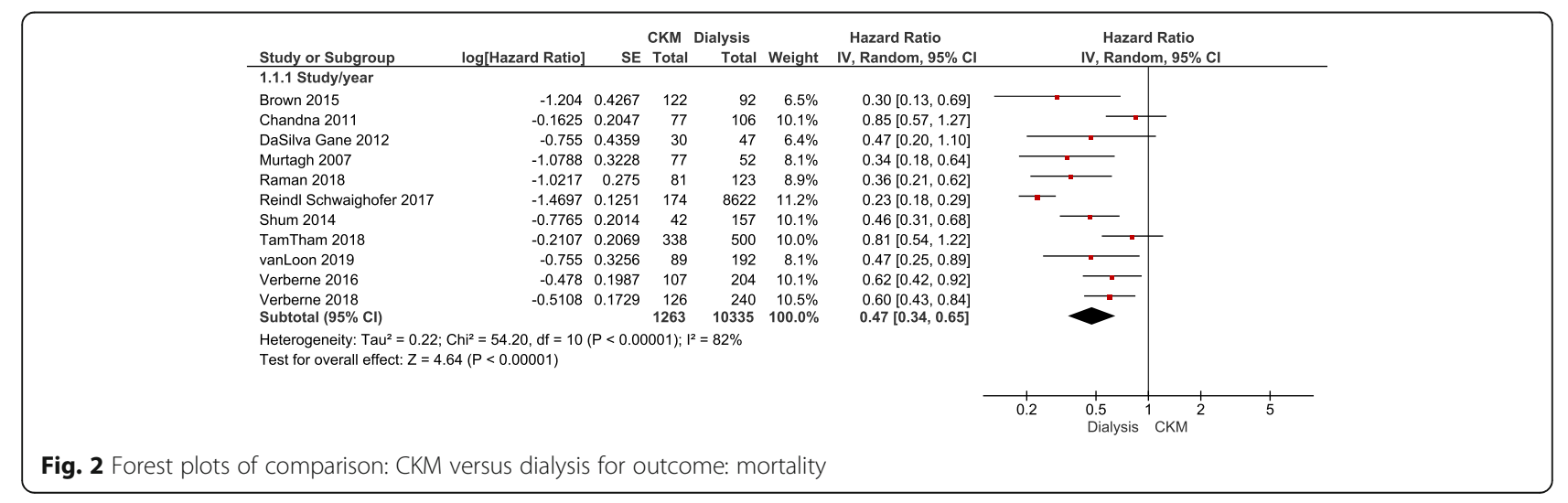


was found over time [29]. Two cross-sectional primary comparative studies found that patients on CKM had a lower symptom burden compared to patients on dialysis [42, 43]. A third cross-sectional study comparing a group of patients with CKD5 receiving CKM and patients on dialysis found overlapping symptom prevalence and intensity between the groups [44].

\section{Hospitalization}

We identified three prospective $[21,22,24]$ and three retrospective [35, 36, 39] primary cohort studies comparing the hospitalization of patients. Results were heterogeneous. Most studies found that patients on CKM had fewer hospital admissions or a significantly lower risk of hospitalization than patients on dialysis [22, 24, 35, 39]. Figure 3 shows a meta-analysis of the number of admission days. One study observed no difference in number of hospital admissions and number of days spent in hospital between groups [21]. Finally, one study comparing patients treated with CKM to patients on PD observed fewer days spent in hospital per person year for the patients treated with PD [36].

\section{Place of death}

Results from two primary studies indicate that patients on CKM more often die at home or in a hospice compared to patients on dialysis, who more often die in hospital $[24,25]$.

\section{Discussion}

\section{Summary of Evidence}

This review identified 25 studies comparing patients with CKD5 choosing or receiving CKM with those choosing or receiving dialysis to investigate the outcomes of mortality, QoL, symptom burden, hospitalization, and place of death. The studies were of variable quality, and there was substantial heterogeneity in presentation of the data, making it difficult to conduct an adequate meta-analysis for most outcomes.

Based on the available evidence, according to our review, CKM does not provide the same or extended survival in patients with CKD5 compared to dialysis. This is in line with previous systematic reviews [9, 12]. Overall, in contrast to CKM, dialysis is life prolonging. However, some studies indicate that the two treatment strategies may provide equal rates of mortality for patients who are 80 years old and above, or elderly patients with high comorbidities [21, 31, 32]. Thus, information on CKM may be considered in clinical practice in relation to this patient group. Some studies indicate that CKM may result in higher QoL compared to dialysis, and patients who receive CKM seem to have less hospitalization than patients on dialysis [21, 41-43]. Regarding symptom burden, results were conflicting. A recently published systematic review [45] concludes that in selected older patients, CKM has the potential to achieve similar QoL compared to a dialysis pathway [45].

Most of the studies identified compared patients on HD with those treated with PD or did not report details of dialysis modality. There was very limited data comparing patients on CKM with patients on PD. Our findings suggest that there may be differences between these two patient groups for the outcomes of symptom burden and hospitalization. Thus, these aspects should be considered in the decision-making process involved in choosing a patient's preferred treatment strategy.

Only one study investigated whether preferred place of death for patients with CKD5 on either CKM or a dialysis pathway was congruent with their actual place of death [24]. The study indicated that patients on the CKM pathway more often die at home or in a hospice compared to patients on dialysis, who more often die in hospital. Studies of the general public and of patients with cancer have shown that most people would prefer to die at home [46, 47]. Based on our review, dying at home or in a hospice seems more likely to be the outcome for patients managed conservatively compared to patients on dialysis $[24,25]$. Whether this result fulfils patients' preferences was, however, unclear.

\section{Strengths and Limitations}

This review was conducted rigorously, using robust processes and relevant software tools. However, the study does have some limitations. All previous studies analysed were of observational design with variable sample size and quality and investigated patient groups that were heterogeneously defined. Furthermore, outcomes were assessed over different time periods. The quality of studies was reduced due to lead time bias when estimating mortality, and by confounders mostly favouring dialysis. Data heterogeneity restricted the use of meta-analysis. A high heterogeneity $\left(\mathrm{I}^{2}=82 \%\right)$ was found in the meta-

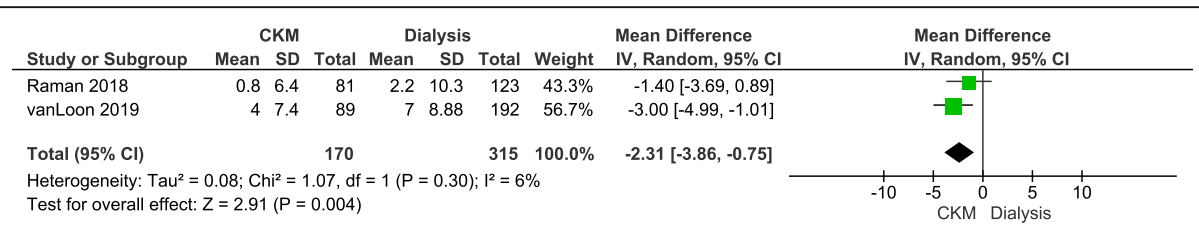

Fig. 3 Forest plot of comparison: CKM versus dialysis for outcome: hospitalization - number of days spent in hospital 
analysis of mortality (See Fig. 2). One reason for this heterogeneity could be variation between countries. The total of included studies represents only 10 countries with more than a third of the studies having been conducted in UK. A sub-analysis of four studies from UK $[3,25,31,40]$ had a moderate heterogeneity $\left(\mathrm{I}^{2}=68 \%\right)$ with a pooled hazard ratio of mortality of $0.49 ; 95 \%$ confidence interval $0.29-0.74$. A sub-analysis of three studies from the Netherlands [21, 32, 33] had a low heterogeneity $\left(\mathrm{I}^{2}=0 \%\right)$ with a pooled hazard ratio of 0.59 ; $95 \%$ confidence interval $0.46-0.74$. A study determining the practice patterns of CKM in UK showed that CKM is acknowledged in all renal units. However, considerable variation was seen in how units described and delivered CKM [48]. We identified such variation also among the studies included in this review which may have caused selection bias and may have in some studies influenced the results in predictable way.

\section{Implications for Clinical Practice and Further Research}

Discussion of treatment options with clinicians is crucial for patients with CKD5 regardless of their preferred modality of treatment. In a recent qualitative study from the UK, 20 patients receiving CKM were interviewed [49]. The patients' experience was that clinicians avoided talking about diagnosis and prognosis related to their disease. The patients expressed a desire to receive information related to their disease and possible treatment choices. At the same time, however, they were ambivalent about receiving detailed knowledge on the progression of their disease. Although the evidence in our review relies on observational data, the results suggest a CKM pathway can be an acceptable alternative to dialysis for patients aged 80 years and above or elderly patients with comorbidities. Consequently, discussion of CKM as a future treatment modality with this group of patients is important. A Canadian survey from 2010 showed that $60 \%$ of the patients receiving dialysis regretted having started the treatment [50]. The findings of one qualitative review indicated that patients with CKD were capable of prioritizing QoL and freedom over survival [51]. Based on the findings of this review, aspects of QoL, symptom burden, and hospitalization should be considered in the decision-making process when choosing the preferred treatment strategy.

To date, the evidence of outcomes for patients with CKD5 receiving dialysis compared to patients on a CKM pathway has been drawn from observational studies of varying quality, many of which were retrospective. No randomized controlled studies have yet been published in this area. For ethical reasons, conducting such studies may be very problematic or even impossible. Thus, future research may also have to rely mainly on observational studies. Such studies should be carefully planned, with a prospective design and a strict methodology to minimize bias and the influence of confounders. Comparative studies of patients on CKM or PD may provide a more nuanced basis for discussions of future treatment choices for patients with CKD5.

\section{Conclusions}

In this systematic review, we explored studies evaluating CKM as an alternative to dialysis for adult patients with CKD5 in relation to mortality, QoL, hospitalization, symptom burden, and place of death. Overall, patients with CKD5 on CKM have poorer survival compared to patients on dialysis. However, we observed that for patients aged 80 years and above, or elderly patients with severe comorbidities, the improved survival on dialysis over CKM appears to vanish. Despite some inconsistencies, the results suggest CKM has advantages compared to dialysis for the outcomes of QoL, hospitalization, symptom burden, and place of death. These findings should be addressed when discussing future treatment options with patients. More rigorously conducted studies are needed to establish a better base for such a decisionmaking process.

\section{Abbreviations \\ CKD5: Stage 5 chronic kidney disease; CKM: Conservative kidney management; QoL: Quality of life; HD: Haemodialysis; PD: Peritoneal dialysis; AAPD: Assisted automated peritoneal dialysis; RCTs: Randomized controlled trials; PRISMA: Preferred reporting items for systematic reviews and meta- analyses; CKD 1-4: Stage 1-4 chronic kidney disease; PICO: Patient, Intervention, Comparison, Outcome; ROBINS-I: Risk Of Bias In Non- randomized Studies of Interventions tool; GRADE: The Grading of Recommendations Assessment, Development and Evaluation methodology; RevMan5: Review Manager software; SD: Standard deviations; 95 \% Cl: 95 \% confidence Interval; HR: Hazard ratios; RR: Relative risks; OR: Odds ratios (OR)}

\section{Supplementary Information}

The online version contains supplementary material available at https://doi. org/10.1186/s12882-021-02516-6.

\section{Additional file 1:}

Additional file 2:

Additional file 3:

Additional file 4:

Additional file 5:

\section{Acknowledgements}

The authors would like to thank the members of the national clinical guideline reference group for their constructive comments and suggestions during the planning and development of this research work. Representatives were: Jan Rishave, Danish Kidney Association; Lisbet Brandi, Danish Society of Nephrology; Karina Bruun, Danish Society of Renal Nurses; Birthe Thoerring, Danish Society of Palliative Medicine; Ellen Holm, Danish Gerontological Society; and Jette Kolding Kristensen, Danish College of General Practitioners. The authors also wish to thank member of the working group Line 
Staermose Jensen for her work on the national clinical guideline, of which this systematic review is a part.

\section{Authors' contributions}

All authors collected the data and conducted the analyses. Buur LE drafted the manuscript in collaboration with Finderup J. All authors critically reviewed and provided intellectual input to the manuscript. All authors read and approved the final manuscript.

\section{Funding}

The review is part of the preparation of a national clinical guideline, funded by the Danish Health Authority.

\section{Availability of data and materials}

Data sharing is not applicable to this article as no dataset were generated or analysed during the current study.

\section{Declarations}

\section{Ethics approval and consent to participate}

Not applicable.

\section{Consent for publication}

Not applicable.

\section{Competing interests}

The authors declare that they have no competing interests.

\section{Author details}

${ }^{1}$ Department of Renal Medicine, Aarhus University Hospital, Palle Juul-Jensens Boulevard 99, 8200 Aarhus, Denmark. ${ }^{2}$ Department of Clinical Medicine, Aarhus University, Aarhus, Denmark. ${ }^{3}$ Department of Public Health, Aarhus University, Aarhus, Denmark. ${ }^{4}$ Department of Palliative Medicine, Rigshospitalet, Copenhagen, Denmark. ${ }^{5}$ Department of Renal Medicine, Herlev and Gentofte Hospital, Herlev, Denmark. ${ }^{6}$ Department of Renal Medicine, Aalborg University Hospital, Aalborg, Denmark. ${ }^{7}$ Department of Medicine, North Zealand Hospital, Hillerød, Denmark.

Received: 1 February 2021 Accepted: 1 September 2021 Published online: 11 September 2021

\section{References}

1. O'Halloran P, Noble H, Norwood K, Maxwell P, Shields J, Fogarty D, et al. Advance Care Planning With Patients Who Have End-Stage Kidney Disease: A Systematic Realist Review. Journal of pain and symptom management 2018;56(5):795-807.e18

2. Thiesson H, Heaf J, Sørensen SS, Povlsen J, Løkkegaard N, Dieperink H, et al. Danish Nephrology Registry (DNR) - Annual Report 2017 [Available from: http://nephrology.dk/wp-content/uploads/2018/10/\%C3 \%85rsrapport-2017. pdf.

3. Da Silva-Gane M, Wellsted D, Greenshields H, Norton S, Chandna SM, Farrington K. Quality of life and survival in patients with advanced kidney failure managed conservatively or by dialysis. Clinical journal of the American Society of Nephrology: CJASN. 2012;7(12):2002-9.

4. Services AH. Conservative Kidney Management 2016 [cited 28-01-2021. Available from: https://www.ckmcare.com/.

5. Grimshaw J, Eccles M, Russell I. Developing clinically valid practice guidelines. Journal of evaluation in clinical practice. 1995:1(1):37-48.

6. Willis K, Cheung M, Slifer S. KDIGO 2012 Clinical Practice Guideline for the Evaluation and Management of Chronic Kidney Disease. Official Journal of the International Society of Nephrology. January 2013;3(1).

7. Farrington K, Covic A, Nistor I, Aucella F, Clyne N, De Vos L, et al. Clinical Practice Guideline on management of older patients with chronic kidney disease stage $3 b$ or higher (eGFR $<45 \mathrm{~mL} / \mathrm{min} / 1.73 \mathrm{~m} 2$ ): a summary document from the European Renal Best Practice Group. Nephrology, dialysis, transplantation: official publication of the European Dialysis and Transplant Association - European Renal Association. 2017:32(1):9-16.

8. National, Guideline, Centre. NICE guideline NG107: RRT and conservative management Modalities of RRT. National Institute for Health and Care Excellence. 2018
9. Foote C, Kotwal S, Gallagher M, Cass A, Brown M, Jardine M. Survival outcomes of supportive care versus dialysis therapies for elderly patients with end-stage kidney disease: A systematic review and meta-analysis. Nephrology (Carlton, Vic). 2016;21(3):241-53.

10. Tsai HB, Chao CT, Chang RE, Hung KY, Group CS. Conservative management and health-related quality of life in end-stage renal disease: a systematic review. Clinical and investigative medicine Medecine clinique et experimentale. 2017;40(3):E127-e34.

11. Vega-Alava KM, Luz VA. A Comparison Between Dialysis Versus Conservative Management as Modes of Treatment in the Management of Elderly Patients with End Stage Renal Disease: A Systematic Review. Philippine Journal of Internal Medicine. 2016;54(4):1-6.

12. Wongrakpanich $S$, Susantitaphong $P$, Isaranuwatchai $S$, Chenbhanich J, Eiam-Ong S, Jaber BL. Dialysis Therapy and Conservative Management of Advanced Chronic Kidney Disease in the Elderly: A Systematic Review. Nephron. 2017;137(3):178-89.

13. Cochrane. Cochrane Handbook for Systematic Reviews of Interventions [Available from: https://training.cochrane.org/handbook.

14. Preferred Reporting Items for Systematic Reviews and Meta-Analyses (PRISMA) website 2015 [cited 02-03-2020. Available from: http://www. prisma-statement.org/.

15. Liberati A, Altman DG, Tetzlaff J, Mulrow C, Gotzsche PC, loannidis JP, et al. The PRISMA statement for reporting systematic reviews and meta-analyses of studies that evaluate health care interventions: explanation and elaboration. Journal of clinical epidemiology. 2009;62(10):e1-34

16. Covidence - Better Systematic Review Management [cited 03-03-2020. Available from: https://www.covidence.org/home.

17. group Gw. GRADE [cited 17-03-2020. Available from: https://www.gra deworkinggroup.org/.

18. RevMan 5 [cited 03-03-2020. Available from: https://community.cochrane. org/help/tools-and-software/revman-5.

19. DerSimonian $\mathrm{R}$, Laird N. Meta-analysis in clinical trials revisited. Contemp Clin Trials. 2015;45(Pt A):139-45

20. O'Connor NR, Kumar P. Conservative management of end-stage renal disease without dialysis: a systematic review. Journal of palliative medicine. 2012:15(2):228-35.

21. van Loon IN, Goto NA, Boereboom FTJ, Verhaar MC, Bots ML, Hamaker ME. Quality of life after the initiation of dialysis or maximal conservative management in elderly patients: a longitudinal analysis of the Geriatric assessment in OLder patients starting Dialysis (GOLD) study. BMC nephrology. 2019;20(1):108.

22. Raman M, Middleton RJ, Kalra PA, Green D. Outcomes in dialysis versus conservative care for older patients: A prospective cohort analysis of stage 5 Chronic Kidney Disease. PLoS One. 2018;13(10):e0206469.

23. Joly D, Anglicheau D, Alberti C, Nguyen AT, Touam M, Grunfeld JP, et al. Octogenarians reaching end-stage renal disease: cohort study of decisionmaking and clinical outcomes. J Am Soc Nephrol. 2003;14(4):1012-21.

24. Carson RC, Juszczak M, Davenport A, Burns A. Is maximum conservative management an equivalent treatment option to dialysis for elderly patients with significant comorbid disease? Clinical journal of the American Society of Nephrology: CJASN. 2009;4(10):1611-9.

25. Smith C, Da Silva-Gane M, Chandna S, Warwicker P, Greenwood R Farrington K. Choosing not to dialyse: evaluation of planned non-dialytic management in a cohort of patients with end-stage renal failure. Nephron Clinical practice. 2003:95(2):c40-6.

26. Teo BW, Ma V, Xu H, Li J, Lee EJ. Profile of hospitalisation and death in the first year after diagnosis of end-stage renal disease in a multi-ethnic Asian population. Annals of the Academy of Medicine, Singapore. 2010;39(2):79-87.

27. Tan T, Brennan F, Brown MA. Impact of dialysis on symptom burden and functional state in the elderly. Renal Society of Australasia Journal. March, 2017;13(1):22-30.

28. Yuen S, Suen HP, Kwok O, Yong S, Tse M. Advance care planning for 600 Chinese patients with end-stage renal disease. Hong Kong Journal of Nephrology. 2016;19:19-27.

29. Brown MA, Collett GK, Josland EA, Foote C, Li Q, Brennan FP. CKD in elderly patients managed without dialysis: survival, symptoms, and quality of life. Clinical journal of the American Society of Nephrology: CJASN. 2015;10(2): 260-8.

30. Seow YY, Cheung YB, Qu LM, Yee AC. Trajectory of quality of life for poor prognosis stage 5D chronic kidney disease with and without dialysis. American journal of nephrology. 2013;37(3):231-8. 
31. Murtagh FE, Marsh JE, Donohoe P, Ekbal NJ, Sheerin NS, Harris FE. Dialysis or not? A comparative survival study of patients over 75 years with chronic kidney disease stage 5. Nephrology, dialysis, transplantation: official publication of the European Dialysis and Transplant Association - European Renal Association. 2007;22(7):1955-62.

32. Verberne WR, Geers AB, Jellema WT, Vincent $H H$, van Delden JJ, Bos WJ. Comparative Survival among Older Adults with Advanced Kidney Disease Managed Conservatively Versus with Dialysis. Clinical journal of the American Society of Nephrology: CJASN. 2016;11(4):633-40.

33. Verberne WR, Dijkers J, Kelder JC, Geers ABM, Jellema WT, Vincent HH, et al. Value-based evaluation of dialysis versus conservative care in older patients with advanced chronic kidney disease: a cohort study. BMC nephrology. 2018;19(1):205.

34. Teruel JL, Burguera Vion V, Gomis Couto A, Rivera Gorrin M, FernandezLucas M, Rodriguez Mendiola N, et al. Choosing conservative therapy in chronic kidney disease. Nefrologia: publicacion oficial de la Sociedad Espanola Nefrologia. 2015;35(3):273-9.

35. Tam-Tham H, Quinn RR, Weaver RG, Zhang J, Ravani P, Liu P, et al. Survival among older adults with kidney failure is better in the first three years with chronic dialysis treatment than not. Kidney international. 2018;94(3):582-8.

36. Shum CK, Tam KF, Chak WL, Chan TC, Mak YF, Chau KF. Outcomes in older adults with stage 5 chronic kidney disease: comparison of peritoneal dialysis and conservative management. The journals of gerontology Series A, Biological sciences and medical sciences. 2014;69(3):308-14.

37. Reindl-Schwaighofer R, Kainz A, Kammer M, Dumfarth A, Oberbauer R. Survival analysis of conservative vs. dialysis treatment of elderly patients with CKD stage 5. PLoS One. 2017;12(7):e0181345.

38. Kwok WH, Yong SP, Kwok OL. Outcomes in elderly patients with end-stage renal disease: Comparison of renal replacement therapy and conservative management. Hong Kong Journal of Nephrology. 2016;19:42-56.

39. Hussain JA, Mooney A, Russon L. Comparison of survival analysis and palliative care involvement in patients aged over 70 years choosing conservative management or renal replacement therapy in advanced chronic kidney disease. Palliative medicine. 2013;27(9):829-39.

40. Chandna SM, Da Silva-Gane M, Marshall C, Warwicker P, Greenwood RN, Farrington K. Survival of elderly patients with stage 5 CKD: comparison of conservative management and renal replacement therapy. Nephrology, dialysis, transplantation: official publication of the European Dialysis and Transplant Association - European Renal Association. 2011;26(5):1608-14.

41. Iyasere O, Brown EA, Johansson L, Davenport A, Farrington K, Maxwell $A P$, et al. Quality of life with conservative care compared with assisted peritoneal dialysis and haemodialysis. Clinical kidney journal. 2019;12(2): 262-8.

42. Almutary $\mathrm{H}$, Bonner A, Douglas $\mathrm{C}$. Which patients with chronic kidney disease have the greatest symptom burden? A comparative study of advanced CKD stage and dialysis modality. Journal of renal care. 2016;42(2): 73-82.

43. Shah KK, Murtagh FEM, McGeechan K, Crail S, Burns A, Tran AD, et al. Health-related quality of life and well-being in people over 75 years of age with end-stage kidney disease managed with dialysis or comprehensive conservative care: a cross-sectional study in the UK and Australia. BMJ open. 2019;9(5):e027776.

44. Yong DS, Kwok AO, Wong DM, Suen MH, Chen WT, Tse DM. Symptom burden and quality of life in end-stage renal disease: a study of 179 patients on dialysis and palliative care. Palliative medicine. 2009;23(2):111-9.

45. Verberne WR, van den Wittenboer ID, Voorend CGN, Abrahams AC, van Buren M, Dekker FW, et al. Health-related quality of life and symptoms of conservative care versus dialysis in patients with end-stage kidney disease: a systematic review. Nephrology, dialysis, transplantation: official publication of the European Dialysis and Transplant Association - European Renal Association. 2020.

46. Gomes B, Calanzani N, Gysels M, Hall S, Higginson IJ. Heterogeneity and changes in preferences for dying at home: a systematic review. BMC palliative care. 2013;12:7.

47. Gomes B, Higginson IJ, Calanzani N, Cohen J, Deliens L, Daveson BA, et al. Preferences for place of death if faced with advanced cancer: a population survey in England, Flanders, Germany, Italy, the Netherlands, Portugal and Spain. Annals of oncology: official journal of the European Society for Medical Oncology. 2012;23(8):2006-15.

48. Roderick P, Rayner H, Tonkin-Crine S, Okamoto I, Eyles C, Leydon G, et al. Health Services and Delivery Research. A national study of practice patterns in UK renal units in the use of dialysis and conservative kidney management to treat people aged 75 years and over with chronic kidney failure. Southampton (UK): NIHR Journals Library

49. Selman LE, Bristowe K, Higginson IJ, Murtagh FEM. The views and experiences of older people with conservatively managed renal failure: a qualitative study of communication, information and decision-making. BMC nephrology. 2019;20(1):38.

50. Davison SN. End-of-life care preferences and needs: perceptions of patients with chronic kidney disease. Clinical journal of the American Society of Nephrology: CJASN. 2010;5(2):195-204.

51. Tong A, Cheung KL, Nair SS, Kurella Tamura M, Craig JC, Winkelmayer WC. Thematic synthesis of qualitative studies on patient and caregiver perspectives on end-of-life care in CKD. American journal of kidney diseases: the official journal of the National Kidney Foundation. 2014;63(6):913-27.

\section{Publisher's Note}

Springer Nature remains neutral with regard to jurisdictional claims in published maps and institutional affiliations.
Ready to submit your research? Choose BMC and benefit from:

- fast, convenient online submission

- thorough peer review by experienced researchers in your field

- rapid publication on acceptance

- support for research data, including large and complex data types

- gold Open Access which fosters wider collaboration and increased citations

- maximum visibility for your research: over $100 \mathrm{M}$ website views per year

At BMC, research is always in progress.

Learn more biomedcentral.com/submissions 